\title{
FAILURE OF THE $L^{1}$ POINTWISE AND MAXIMAL ERGODIC THEOREMS FOR THE FREE GROUP
}

\author{
TERENCE TAO \\ UCLA Department of Mathematics, Los Angeles, CA 90095-1555, USA; \\ email: tao@math.ucla.edu
}

Received 17 May 2015; accepted 1 November 2015

\begin{abstract}
Let $F_{2}$ denote the free group on two generators $a$ and $b$. For any measure-preserving system $\left(X, \mathcal{X}, \mu,\left(T_{g}\right)_{g \in F_{2}}\right)$ on a finite measure space $X=(X, \mathcal{X}, \mu)$, any $f \in L^{1}(X)$, and any $n \geqslant 1$, define the averaging operators

$$
\mathcal{A}_{n} f(x):=\frac{1}{4 \times 3^{n-1}} \sum_{g \in F_{2}:|g|=n} f\left(T_{g}^{-1} x\right),
$$

where $|g|$ denotes the word length of $g$. We give an example of a measure-preserving system $X$ and an $f \in L^{1}(X)$ such that the sequence $\mathcal{A}_{n} f(x)$ is unbounded in $n$ for almost every $x$, thus showing that the pointwise and maximal ergodic theorems do not hold in $L^{1}$ for actions of $F_{2}$. This is despite the results of Nevo-Stein and Bufetov, who establish pointwise and maximal ergodic theorems in $L^{p}$ for $p>1$ and for $L \log L$ respectively, as well as an estimate of Naor and the author establishing a weak-type $(1,1)$ maximal inequality for the action on $\ell^{1}\left(F_{2}\right)$. Our construction is a variant of a counterexample of Ornstein concerning iterates of a Markov operator.
\end{abstract}

2010 Mathematics Subject Classification: 37A30 (primary)

\section{Introduction}

Let $F_{2}$ denote the free non-Abelian group on two generators $a$ and $b$. Define a reduced word to be a word with letters in the alphabet $\left\{a, b, a^{-1}, b^{-1}\right\}$ in which $a, a^{-1}$ and $b, b^{-1}$ are never adjacent, and for each $g \in F_{2}$, define the word length $|g|$ of $g$ to be the length of the unique reduced word that produces $g$. We let $F_{2}^{2}$ denote the index 2 subgroup of $F_{2}$ consisting of $g \in F_{2}$ with even word length.

(c) The Author 2015. This is an Open Access article, distributed under the terms of the Creative Commons Attribution licence (http://creativecommons.org/licenses/by/4.0/), which permits unrestricted re-use, distribution, and reproduction in any medium, provided the original work is properly cited. 
Define an $F_{2}$-system to be a quadruple $\left(X, \mathcal{X}, \mu,\left(T_{g}\right)_{g \in F_{2}}\right)$, where $(X, \mathcal{X}, \mu)$ is a measure space with $0<\mu(X)<\infty$, and $T_{g}: X \rightarrow X$ is a family of measurepreserving maps on $X$ for $g \in F_{2}$, with $T_{1}$ the identity and $T_{g} T_{h}=T_{g h}$ for all $g, h \in F_{2}$; in particular, $T_{g}$ are bimeasurable with $T_{g}^{-1}=T_{g^{-1}}$. One can of course normalize such systems to have total measure 1 by dividing $\mu$ by $\mu(X)$, but (as we will eventually be gluing several systems together) it will be convenient not to always insist on such a normalization. As the free group $F_{2}$ has no relations, such a system can be prescribed by specifying two arbitrary invertible bimeasurable measure-preserving maps $T_{a}, T_{b}: X \rightarrow X$, and then defining $T_{g}$ for all other $g \in G$ in the obvious manner.

We say that an $F_{2}$-system is $F_{2}$-ergodic if all $F_{2}$-invariant measurable sets either have zero measure or full measure, and $F_{2}^{2}$-ergodic if the same claim is true for $F_{2}^{2}$-invariant measurable sets. For any $f \in L^{1}(X)=L^{1}(X, \mathcal{X}, \mu)$ and any $n \geqslant 1$, we define the averaging operators

$$
\mathcal{A}_{n} f(x):=\frac{1}{4 \times 3^{n-1}} \sum_{g \in F_{2}:|g|=n} f\left(T_{g}^{-1} x\right) ;
$$

note that $4 \times 3^{n-1}$ is the number of reduced words of length $n$. One can of course use symmetry to replace $T_{g}^{-1}$ by $T_{g}$ if desired.

The pointwise convergence of the operators $\mathcal{A}_{n}$ was studied by Nevo and Stein [10] and Bufetov [3], who (among other things) proved the following result.

THEOREM 1 (Pointwise ergodic theorem). Let $\left(X, \mathcal{X}, \mu,\left(T_{g}\right)_{g \in F_{2}}\right)$ be an $F_{2}$ system. If $\int_{X}|f| \log (2+|f|) d \mu<\infty$, then $\mathcal{A}_{2 n} f$ converges pointwise almost everywhere (and in $L^{1}(X)$ norm) to an $F_{2}^{2}$-invariant function. In particular, if $\left(X, \mathcal{X}, \mu,\left(T_{g}\right)_{g \in F_{2}}\right)$ is $F_{2}^{2}$-ergodic, then $\mathcal{A}_{2 n} f$ converges pointwise almost everywhere and in $L^{1}$ to the constant $(1 / \mu(X)) \int_{X} f d \mu$.

The restriction to even averages $\mathcal{A}_{2 n}$, and the use of $F_{2}^{2}$ instead of $F_{2}$, can be seen to be necessary by considering the simple example in which $X$ is a twoelement set $\{0,1\}$ (with uniform measure) and $T_{a}$ and $T_{b}$ interchange the two elements 0 and 1 of this set. The original paper of Nevo and Stein [10] established this theorem for $f \in L^{p}(X)$ for some $p>1$, by modifying the methods of Stein [14]. The subsequent paper of Bufetov [3] used instead the 'Alternierende Verfahren' of Rota [13] to cover the $L \log L$ case. Both arguments also extend to several other group actions (see for example $[6,8,11]$ ), but for simplicity of exposition we shall focus only on the $F_{2}$ case. Furthermore, we remark that both arguments also give bounds on the associated maximal operator $f \mapsto \sup _{n} \mathcal{A}_{n}|f|$. See also $[1,2]$ for an alternate approach to pointwise ergodic theorems in $L^{p}$ and $L \log L$. 
In [10] the question was posed as to whether the above pointwise ergodic theorem extended to arbitrary $L^{1}(X)$ functions. The main result of this paper answers this question in the negative.

THEOREM 2 (Counterexample). There exist an $F_{2}$-system $\left(X, \mathcal{X}, \mu,\left(T_{g}\right)_{g \in F_{2}}\right)$ and a nonnegative function $f \in L^{1}(X)$ such that $\sup _{n}\left|\mathcal{A}_{2 n} f(x)\right|=\infty$ for almost every $x \in X$. In particular, $\mathcal{A}_{2 n} f(x)$ fails to converge to a finite limit as $n \rightarrow \infty$ for almost every $x \in X$.

As such, there is no pointwise ergodic theorem or maximal ergodic theorem in $L^{1}$ for actions of the free group $F_{2}$. Our construction also applies to free groups $F_{r}$ on $r$ generators for any $r \geqslant 2$; we leave the modification of the arguments below to this more general case to the interested reader. This result stands in contrast to the situation for the regular action of $F_{2}$ on $\ell^{1}\left(F_{2}\right)$, for which a weak-type $(1,1)$ for the maximal operator was established by Naor and the author [9, Theorem 1.5]. Note that the estimate for $\ell^{1}\left(F_{2}\right)$ does not transfer to arbitrary $F_{2}$-systems due to the nonamenability of the free group $F_{2}$.

Because the sphere $\left\{g \in F_{2}:|g|=n\right\}$ is a positive fraction of the ball $\left\{g \in F_{2}\right.$ : $|g| \leqslant n\}$, the above result also holds if the average over spheres is replaced with an average over balls, or with regard to other minor variations of the spherical averaging operator such as $\frac{1}{2} \mathcal{A}_{n}+\frac{1}{2} \mathcal{A}_{n+1}$. This negative result for averaging on balls stands in contrast with the situation for amenable groups, for which pointwise and maximal ergodic results in $L^{1}$ are established for suitable replacements of balls, such as tempered Følner sets; see [7]. On the other hand, if one considers the Cesàro means $(1 / N) \sum_{n \leqslant N} \mathcal{A}_{n}$ of spherical averages on $F_{2}$-systems, then pointwise and maximal ergodic theorems in $L^{1}$ were established in [10]. Combining these theorems with Theorem 2, we see that the averages $\mathcal{A}_{2 n} f(x)$ do not diverge to infinity almost everywhere (because their averages converge almost everywhere to a finite limit), but simply do not have a limit at all. See also [4-6] for further ergodic theorems for Cesàro means in other groups than the free group.

Our construction is inspired by a well-known counterexample of Ornstein [12] demonstrating the failure of the maximal ergodic theorem in $L^{1}$ for iterates $P^{n}$ of a certain well-chosen self-adjoint Markov operator. Roughly speaking, the function $f$ in Ornstein's example consists of many components $f_{i}$, each of which comes with a certain 'time delay' that ensures that the dynamics of $P^{n} f_{i}$ only become significant after a considerable period of time-in particular, long enough for the dynamics of other components of the function to have achieved 'mixing' in the portion of $X$ where the most interesting part of the dynamics of $P^{n} f_{i}$ takes place, allowing the amplitude of $f_{i}$ to be slightly smaller than would otherwise 
have been necessary to make $\sup _{n} P^{n} f$ large. To adapt this construction to the setting of $F_{2}$-systems, we need to glue together various $F_{2}$-systems that have the capability to produce such a 'time delay'. We will be able to construct such systems by basically taking an 'infinitely large ball' in $F_{2}$, gluing the boundary of that ball to itself, and redefining the shift maps on the boundary appropriately. Somewhat ironically, the positive results in Theorem 1 play a helpful supporting role in establishing the negative result in Theorem 2, by establishing the 'mixing' referred to previously, which is an essential part of Ornstein's construction.

\section{Initial reductions}

We begin by reducing Theorem 2 to the following more quantitative statement.

THEOREM 3 (Quantitative counterexample). Let $\alpha, \varepsilon>0$. Then there exist an $F_{2}$-system $\left(X, \mathcal{X}, \mu,\left(T_{g}\right)_{g \in F_{2}}\right)$ and a nonnegative function $f \in L^{\infty}(X)$, such that

$$
\|f\|_{L^{1}(X)} \leqslant \alpha \mu(X)
$$

but such that

$$
\sup _{n} \mathcal{A}_{2 n} f(x) \geqslant 1-\varepsilon
$$

for all $x \in X$ outside of a set of measure at most $\varepsilon \mu(X)$.

Let us see how Theorem 3 implies Theorem 2. By dividing $\mu$ by $\mu(X)$ we may normalize $\mu(X)=1$ in Theorem 3. Applying the above theorem with $\alpha=\varepsilon=$ $2^{-m}$, we can thus find for each natural number $m$, an $F_{2}$-system $\left(X_{m}, \mathcal{X}_{m}, \mu_{m}\right.$, $\left.\left(T_{g, m}\right)_{g \in F_{2}}\right)$ with $\mu_{m}\left(X_{m}\right)=1$, and a nonnegative function $f_{m} \in L^{\infty}\left(X_{m}\right)$ such that

$$
\left\|f_{m}\right\|_{L^{1}\left(X_{m}\right)} \leqslant 2^{-m}
$$

and

$$
\sup _{n} \mathcal{A}_{2 n} f_{m}(x) \geqslant 1-2^{-m}
$$

outside of a set of measure $2^{-m}$.

Let $\left(X, \mathcal{X}, \mu,\left(T_{g}\right)_{g \in F_{2}}\right)$ be the product system; thus $X$ is the Cartesian product $X:=\prod_{m} X_{m}$ with product $\sigma$-algebra $\mathcal{X}:=\prod_{m} \mathcal{X}_{m}$, product probability measure $\mu:=\prod_{m} \mu_{m}$, and product action $T_{g}:=\biguplus_{m} T_{g, m}$. Each $f_{m} \in L^{\infty}\left(X_{m}\right)$ then lifts to a function $\tilde{f}_{m} \in L^{\infty}(X)$ with

$$
\left\|\tilde{f}_{m}\right\|_{L^{1}(X)} \leqslant 2^{-m}
$$

and

$$
\sup _{n} \mathcal{A}_{2 n} \tilde{f}_{m}(x) \geqslant 1-2^{-m} \geqslant 1 / 2
$$


outside of a set of measure $2^{-m}$. If we then set $f:=\sum_{m} m \tilde{f}_{m}$, then $f \in L^{1}(X)$, and from the pointwise inequality

$$
\sup _{n} \mathcal{A}_{2 n} f(x) \geqslant m_{0} \sup _{n} \mathcal{A}_{2 n} \tilde{f}_{m}(x)
$$

for all $m \geqslant m_{0}$ and the Borel-Cantelli lemma we see that $\sup _{n} \mathcal{A}_{2 n} f(x)$ is larger than $m_{0} / 2$ for almost every $x$ and any given $m_{0}$, which yields the claim.

It remains to prove Theorem 3. In order to adapt the arguments of Ornstein [12], we would like to interpret the averaging operators $\mathcal{A}_{n}$ as powers $P^{n}$ of a Markov operator $P$. This is not true as stated, since we do not quite have the semigroup property $\mathcal{A}_{n} \mathcal{A}_{m}=\mathcal{A}_{n+m}$ (although $\mathcal{A}_{n} \mathcal{A}_{m}$ does contain a term of the form $\frac{3}{4} \mathcal{A}_{n+m}$ ). However, as observed by Bufetov [3], we can recover a Markov interpretation for $\mathcal{A}_{n}$ by lifting $X$ up to a four-fold cover $\tilde{X}$ that tracks the 'outward normal vector' for the sphere. More precisely, given an $F_{2}$-system $(X, \mathcal{X}, \mu$, $\left.\left(T_{g}\right)_{g \in F_{2}}\right)$, we define the lifted measure space $(\tilde{X}, \tilde{\mathcal{X}}, \tilde{\mu})$ to be the product of $(X, \mathcal{X}, \mu)$ and the four-element space $\left\{a, b, a^{-1}, b^{-1}\right\}$ with the uniform probability measure; in particular, $\tilde{\mu}(\tilde{X})=\mu(X)$. Let $\pi: \tilde{X} \rightarrow X$ be the projection operator $\pi(x, s):=x$; this induces a pushforward operator $\pi_{*}: L^{1}(\tilde{X})$ $\rightarrow L^{1}(X)$ and a pullback operator $\pi^{*}: L^{1}(X) \rightarrow L^{1}(\tilde{X})$ by the formulas

$$
\pi_{*} \tilde{f}(x):=\frac{1}{4} \sum_{s \in\left\{a, b, a^{-1}, b^{-1}\right\}} \tilde{f}(x, s)
$$

and

$$
\pi^{*} f(x, s):=f(x)
$$

for $f \in L^{1}(X)$ and $\tilde{f} \in L^{1}(\tilde{X})$. We also define the Markov operator $P: L^{1}(\tilde{X}) \rightarrow$ $L^{1}(\tilde{X})$ by

$$
P \tilde{f}(x, s):=\frac{1}{3} \sum_{s^{\prime} \in\left\{a, b, a^{-1}, b^{-1}\right\}: s^{\prime} \neq s^{-1}} \tilde{f}\left(T_{s}^{-1} x, s^{\prime}\right) .
$$

One can view $P$ as the Markov operator associated with the Markov chain that for each unit time, moves a given point $(x, s)$ of $\tilde{X}$ to one of the three points $\left(T_{s}^{-1} x\right.$, $s^{\prime}$ ) with $s^{\prime} \in\left\{a, b, a^{-1}, b^{-1}\right\} \backslash\left\{s^{-1}\right\}$, chosen uniformly at random; the adjoint of this chain (which controls how $P f$ propagates the support of $f$ ) maps $(x, s)$ to $\left(T_{s^{\prime}} x, s^{\prime}\right)$ with $s^{\prime} \in\left\{a, b, a^{-1}, b^{-1}\right\} \backslash\left\{s^{-1}\right\}$ drawn uniformly at random. By writing the elements of $\left\{g \in F_{2}:|g|=n\right\}$ as reduced words of length $n$, one can easily verify the identity

$$
\mathcal{A}_{n} f=\pi_{*} P^{n} \pi^{*} f
$$

for any $f \in L^{1}(X)$ and $n \geqslant 1$. It thus suffices to show the following theorem. 
THEOREM 4 (Quantitative counterexample, again). Let $\alpha, \varepsilon>0$. Then there exist an $F_{2}$-system $\left(X, \mathcal{X}, \mu,\left(T_{g}\right)_{g \in F_{2}}\right)$ and a nonnegative function $\tilde{f} \in L^{\infty}(\tilde{X})$, such that

$$
\|\tilde{f}\|_{L^{1}(\tilde{X})} \leqslant \alpha \mu(X)
$$

but such that

$$
\sup _{n} \pi_{*} P^{2 n} \tilde{f}(x) \geqslant 1-\varepsilon
$$

for all $x \in X$ outside of a set of measure at most $\varepsilon \mu(X)$.

Indeed, by setting $f:=4 \pi_{*} \tilde{f}$, and noting the pointwise bound $\tilde{f} \leqslant \pi^{*} f$ and the identity $\|f\|_{L^{1}(X)}=4\|\tilde{f}\|_{L^{1}(\tilde{X})}$, we obtain Theorem 3 (after replacing $\alpha$ by $\alpha / 4$ ).

For inductive reasons, we will prove a technical special case of Theorem 4 , in which the $F_{2}$-system is of a certain 'good' form, and the sequence $\left(P^{n} \tilde{f}\right)_{n \geqslant 0}$ is part of an 'ancient Markov chain' $\left(\tilde{f}_{n}\right)_{n \in \mathbb{Z}}$ that extends to arbitrarily negative times as well as arbitrarily positive times. More precisely, let us define a good system to be an $F_{2}$-system $\left(X, \mathcal{X}, \mu,\left(T_{g}\right)_{g \in F_{2}}\right)$, which admits a decomposition $X=X_{a} \cup X_{b} \cup X_{0}$ into three disjoint sets $X_{a}, X_{b}, X_{0}$ admitting the following (somewhat technical) properties:

(i) (Measure) One has $\mu\left(X_{a}\right)=\mu\left(X_{b}\right)=\frac{1}{4} \mu(X)$ and $\mu\left(X_{0}\right)=\frac{1}{2} \mu(X)$. Furthermore, for any $0 \leqslant \kappa \leqslant \mu\left(X_{b}\right)$, one can find a measurable subset of $X_{b}$ of measure exactly equal to $\kappa$.

(ii) (Invariance) One has $T_{a} X_{a}=X_{a}$ and $T_{b} X_{b}=X_{b}$. In addition, one has the inclusions $T_{a} X_{b} \subset T_{b} X_{a} \cup T_{b}^{-1} X_{a} \subset X_{0}$.

(iii) (Ergodicity) One can partition $X_{a}$ into finitely many $T_{a}$-invariant components $X_{a, 1}, \ldots, X_{a, m}$ of positive measure, such that $T_{a}^{2}$ is ergodic on each of the components $X_{a, i}$; that is, the only $T_{a}^{2}$-invariant measurable subsets of $X_{a, i}$ have measure either 0 or $\mu\left(X_{a, i}\right)$.

(iv) (Generation) One has $X=\bigcup_{g \in F_{2}} T_{g} X_{a, i}$ up to null sets for each $i=1$, $\ldots, m$.

Note that relatively few conditions are required on the dynamics on $X_{b}$; in particular, the ergodicity hypotheses on the system are located in the disjoint region $X_{a}$. This will allow us to easily modify the dynamics on $X_{b}$ in order to 'glue' two good systems together in Section 4.

See Figure 1. We will construct good systems in subsequent sections. For now, we record one useful property of such systems. 


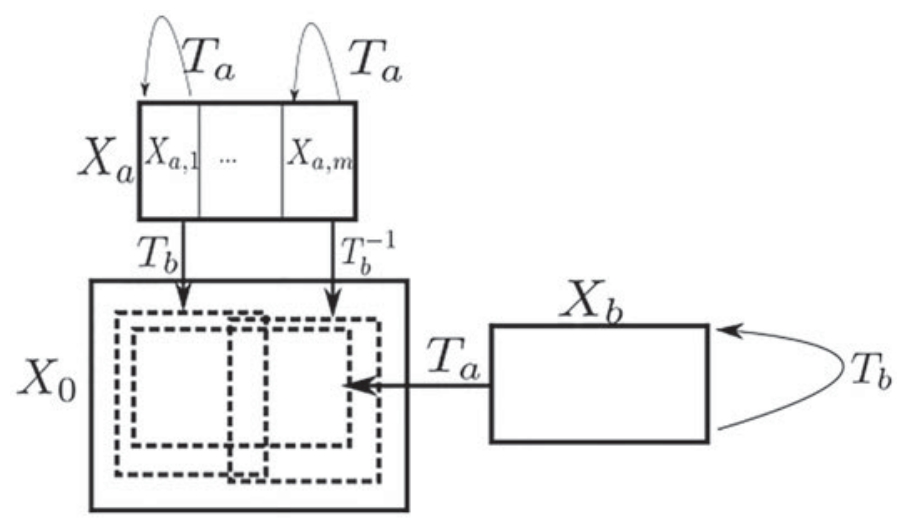

Figure 1. A somewhat schematic depiction of a good system. Only part of the action of $T_{a}$ and $T_{b}$ is displayed.

LEMMA 5 (Pointwise ergodic theorem for good systems). Every good system $\left(X, \mathcal{X}, \mu,\left(T_{g}\right)_{g \in F_{2}}\right)$ is $F_{2}^{2}$-ergodic. In particular (by Theorem 1 ), for any $f \in$ $L^{\infty}(X)$, the averages $\mathcal{A}_{2 n} f$ converge pointwise almost everywhere and in $L^{1}$ norm to $(1 / \mu(X)) \int_{X} f d \mu$. Furthermore, for any $\tilde{f} \in L^{\infty}(\tilde{X}), P^{2 n} \tilde{f}$ converges pointwise almost everywhere and in $L^{1}$ norm to $(1 / \mu(X)) \int_{\tilde{X}} \tilde{f} d \tilde{\mu}$.

Proof. Let $f \in L^{\infty}(X)$ be an $F_{2}^{2}$-invariant function; to establish $F_{2}^{2}$-ergodicity, it will suffice to show that $f$ is constant almost everywhere. As $f$ is $T_{a}^{2}$-invariant, we see from Property (iii) that $f$ is constant almost everywhere on each $X_{a, i}$. Since $F_{2}=F_{2}^{2} \cup F_{2}^{2} a$, we see from Property (iv), the $T_{a}$-invariance of $X_{a, i}$, and the $F_{2}^{2}$-invariance of $f$ that $f$ is constant almost everywhere on $X$, as required. The final claim does not quite follow from Theorem 1 , but is immediate from [3, Proposition 1].

For any $\alpha>0$, let $P(\alpha)$ denote the following claim.

Claim $6(P(\alpha))$. For any $\varepsilon>0$, there exist a good system $\left(X, \mathcal{X}, \mu,\left(T_{g}\right)_{g \in F_{2}}\right)$ with associated decomposition $X=X_{a} \cup X_{b} \cup X_{0}$, and a sequence of nonnegative functions $\tilde{f}_{n} \in L^{\infty}(\tilde{X})$ for $n \in \mathbb{Z}$ with the following properties:

(v) (Ancient Markov chain) $\tilde{f}_{n+1}=P \tilde{f}_{n}$ for all $n \in \mathbb{Z}$. Equivalently, one has $\tilde{f}_{n+m}=P^{m} \tilde{f}_{n}$ for all $n \in \mathbb{Z}$ and $m \in \mathbb{N}$. In particular, $\left\|\tilde{f}_{n}\right\|_{L^{1}(\tilde{X})}$ is independent of $n$.

(vi) (Size) One has $\left\|\tilde{f}_{n}\right\|_{L^{1}(\tilde{X})}=\alpha \mu(X)$ for some $n \in \mathbb{Z}$ (and hence for all $n \in \mathbb{Z})$. 
(vii) (Early support) $\tilde{f}_{n}$ is supported in $\tilde{X}_{0}$ for all negative $n$. Furthermore, there exists a finite $A>0$ such that $\tilde{f}_{n}$ is supported in a set of measure at most $A 3^{n} \mu(X)$ for all negative $n$.

(viii) (Large maximum function) We have

$$
\sup _{n \in \mathbb{Z}} \pi_{*} \tilde{f}_{2 n}(x) \geqslant 1-\varepsilon
$$

for all $x \in X$ outside of a set of measure at most $\varepsilon \mu(X)$.

Note that our sequence $\tilde{f}_{n}$ is ancient in the sense that it extends to arbitrary negative times $n \rightarrow-\infty$ as well as to arbitrary positive times $n \rightarrow \infty$. This will be essential in order to set up suitable 'time delays' in our arguments in later sections. One can informally think of $\tilde{f}_{n}$ as the (normalized) distribution at time $n$ of an ancient Markov process that starts from an infinitely small location deep inside $\tilde{X}_{0}$ at infinite negative time $n=-\infty$, and only escapes $\tilde{X}_{0}$ at or after time $n=0$, and which covers most of $X$ with density roughly 1 or more at some point in time (but crucially, different regions of $X$ may be covered in this manner at different times).

Observe that if $P(\alpha)$ holds for an arbitrarily small set of $\alpha>0$, and $\varepsilon>0$ is arbitrary, then from Properties (vii) and (viii), one has for any $N$ that

$$
\sup _{n \geqslant-N} \pi_{*} f_{2 n}(x) \geqslant 1-\varepsilon
$$

for all $x \in X$ outside of a set of measure at most $\left(\varepsilon+\sum_{n<-N} A 3^{-2 n}\right) \mu(X)$. Taking $N$ large enough (depending on $\varepsilon$ and $A$ ) and setting $\tilde{f}:=\tilde{f}_{-2 N}$, we obtain Theorem 4 (after adjusting $\varepsilon$ as necessary). It thus suffices to show that $P(\alpha)$ holds for arbitrarily small $\alpha>0$. This will be accomplished using the following two key theorems (the second of which is a variant of [12, Lemma 4]).

THEOREM 7 (Initial construction). The claim P(1) is true.

THEOREM 8 (Iteration step). Suppose that $P(\alpha)$ holds for some $0<\alpha \leqslant 1$. Then $P(\alpha(1-\alpha / 4))$ is true.

From Theorems 7 and 8 we see that the infimum of all $0<\alpha \leqslant 1$ for which $P(\alpha)$ holds is zero, and the claim follows. Thus it suffices to establish Theorems 7 and 8 . This will be accomplished in the following two sections.

\section{The initial construction}

We now prove Theorem 7. We will in fact construct an example of a good system $\left(X, \mathcal{X}, \mu,\left(T_{g}\right)_{g \in F_{2}}\right)$ and functions $\tilde{f}_{n}$, which witness $P(1)$ for all $\varepsilon>0$ at once. 
We begin by constructing an appropriate measure space $(X, \mathcal{X}, \mu)$. For each integer $n$, let $Y_{n}$ denote the space of half-infinite reduced words $\left(s_{m}\right)_{m \geqslant n}=$ $s_{n} s_{n+1} s_{n+2} \ldots$, in which each of the $s_{i}$ terms is drawn from the alphabet $\{a$, $\left.b, a^{-1}, b^{-1}\right\}$, and $a, a^{-1}$ and $b, b^{-1}$ are never adjacent. We give this space the product $\sigma$-algebra $\mathcal{Y}_{n}$ (that is, the minimal $\sigma$-algebra for which the coordinate maps $\left(s_{m}\right)_{m \leqslant n} \mapsto s_{m}$ are all measurable). By the Kolmogorov extension theorem, we may construct a probability measure $\mu_{n}$ on $Y_{n}$ such that each finite reduced subword $s_{n} \ldots s_{n+k}$ for $k \geqslant 0$ occurs as an initial segment with probability $1 /\left(4 \times 3^{k}\right)$; one can view this measure as the law of the random half-infinite reduced word constructed by choosing $s_{n}$ uniformly at random from $\{a, b$, $\left.a^{-1}, b^{-1}\right\}$ and then recursively selecting $s_{n+i+1}$ for $i=0,1,2, \ldots$ to be drawn uniformly from $\left\{a, b, a^{-1}, b^{-1}\right\} \backslash\left\{s_{n+i}^{-1}\right\}$.

The disjoint union $Y:=\biguplus_{n \in \mathbb{Z}} Y_{n}$ of $Y_{n}$ admits an action $\left(S_{g}\right)_{g \in F_{2}}$ of $F_{2}$, with the action $S_{s}$ of a generator $s \in\left\{a, b, a^{-1}, b^{-1}\right\}$ defined by setting

$$
S_{s}\left(s_{n} s_{n+1} s_{n+2} \ldots\right):=s s_{n} s_{n+1} s_{n+2} \cdots \in Y_{n-1}
$$

for $s_{n} s_{n+1} s_{n+2} \cdots \in Y_{n}$ and $s \in\left\{a, b, a^{-1}, b^{-1}\right\} \backslash\left\{s_{n}^{-1}\right\}$, and

$$
S_{s}\left(s_{n} s_{n+1} s_{n+2} \ldots\right):=s_{n+1} s_{n+2} \cdots \in Y_{n+1}
$$

for $s_{n} s_{n+1} s_{n+2} \cdots \in Y_{n}$ and $s=s_{n}^{-1}$; thus $S_{g}$ is the operation of formal left multiplication by $g$, after reducing any nonreduced words. If we give $Y$ the measure $\mu_{Y}:=\sum_{n \in \mathbb{Z}} 3^{-n} \mu_{n}$, then one can easily verify that this action is measurepreserving. Unfortunately, $\mu_{Y}$ is an infinite measure due to the contribution of the negative $n$, and so this space is not quite suitable for our needs. Instead, we shall work with a certain subquotient of $Y$, defined as follows.

First, we restrict $Y$ to the space $\biguplus_{n \geqslant 0} Y_{n}=\biguplus_{n \geqslant 1} Y_{n} \uplus Y_{0}$, which can be thought of as a suitably rescaled limit of an 'infinitely large ball' in $F_{2}$, with $Y_{0}$ being the 'boundary' of this ball, and $Y_{n}$ lying increasingly deeper in the 'interior' of the ball as $n$ increases (see Figure 2). This makes the shift maps $S_{s}, s \in\left\{a, b, a^{-1}\right.$, $b^{-1}$ \} partially undefined on the $Y_{0}$ boundary, but we will fix this later by redefining these maps on (a quotient of) $Y_{0}$. Next, we introduce a reflection operation $x \mapsto \bar{x}$ on the boundary $Y_{0}$ by mapping

$$
\overline{s_{0} s_{1} s_{2} \ldots}:=s_{0}^{-1} s_{1}^{-1} s_{2}^{-2} \ldots
$$

It is clear that this map preserves the measure $\mu_{0}$. If we then form the quotient space $Y_{0} / \sim:=\left\{\{x, \bar{x}\}: x \in Y_{0}\right\}$, we can obtain a probability measure $\mu_{0} / \sim$ on $Y_{0} / \sim$ by pushing forward the probability measure $\mu_{0}$ under the quotient map. We observe that $Y_{0} / \sim$ splits into two components of equal measure $1 / 2$, namely $\left(\left(S_{a} Y_{1} \cap Y_{0}\right) \cup\left(S_{a}^{-1} Y_{1} \cap Y_{0}\right)\right) / \sim$ and $\left(\left(S_{b} Y_{1} \cap Y_{0}\right) \cup\left(S_{b}^{-1} Y_{1} \cap Y_{0}\right)\right) / \sim$, noting that 


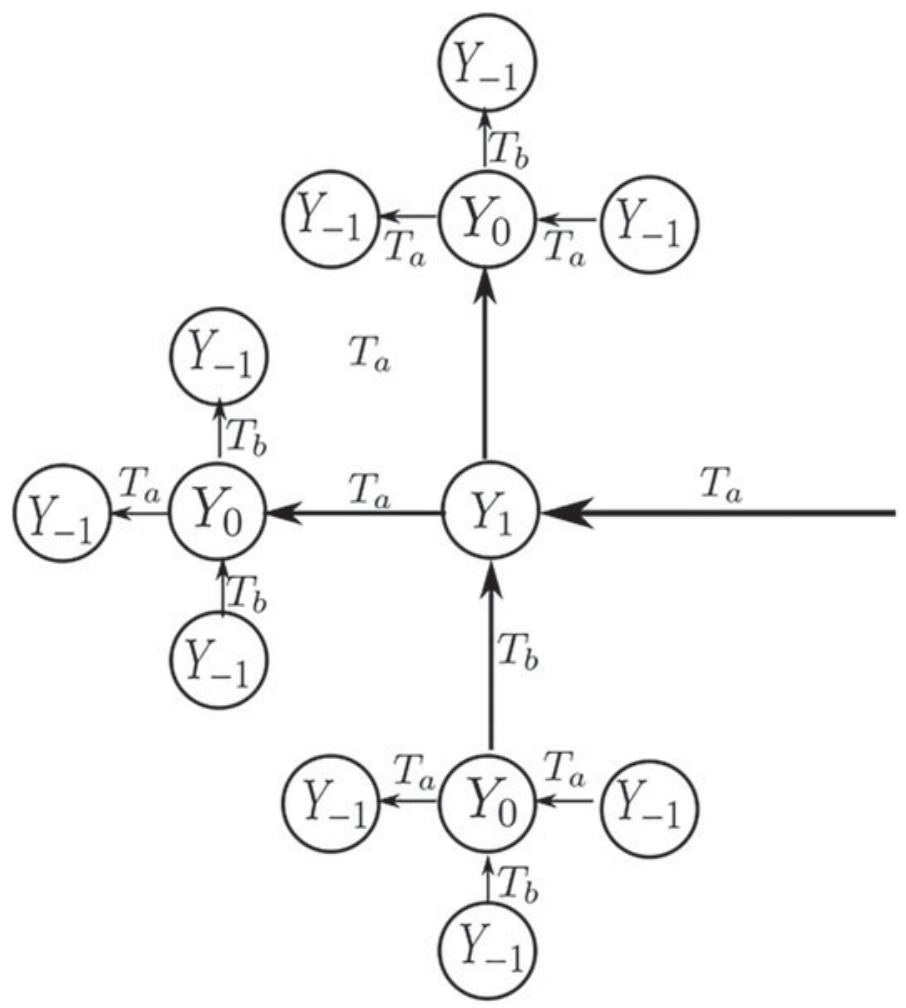

Figure 2. A fragment of the infinite measure space $Y$. The centre disk represents a portion of $Y_{1}$ consisting of reduced words $s_{1} s_{2} \ldots$ with initial letter $s_{1}=a$. The remaining disks are images of this disk under shifts by various elements of $F_{2}$, and all have equal measure with respect to $\mu_{Y}$. This image should be compared with the infinite tree that is the Cayley graph of $F_{2}$.

the sets $S_{a} Y_{1} \cap Y_{0}, S_{a}^{-1} Y_{1} \cap Y_{0}$ are disjoint reflections of each other, and similarly for $S_{b} Y_{1} \cap Y_{0}, S_{b}^{-1} Y_{1} \cap Y_{0}$.

We then define $X$ to be the quotient space $\biguplus_{n \geqslant 1} Y_{n} \uplus\left(Y_{0} / \sim\right)$ with measure $\mu:=\sum_{n \geqslant 1} 3^{-n} \mu_{n}+\frac{1}{2}\left(\mu_{0} / \sim\right)$; thus

$$
\mu(X)=\sum_{n \geqslant 1} 3^{-n}+\frac{1}{2}=1 .
$$

We set $X_{0}:=\biguplus_{n \geqslant 1} Y_{n}, X_{a}:=\left(\left(S_{b} Y_{1} \cap Y_{0}\right) \cup\left(S_{b}^{-1} Y_{1} \cap Y_{0}\right)\right) / \sim$, and $X_{b}:=\left(\left(S_{a} Y_{1} \cap\right.\right.$ $\left.\left.Y_{0}\right) \cup\left(S_{a}^{-1} Y_{1} \cap Y_{0}\right)\right) / \sim$. Thus 


$$
\mu\left(X_{0}\right)=\sum_{n \geqslant 1} 3^{-n}=\frac{1}{2}
$$

and $\mu\left(X_{a}\right)=\mu\left(X_{b}\right)=\frac{1}{4}$. One can think of $X_{0}$ as the 'interior' of $X$, with $X_{a}$ and $X_{b}$ being two equally sized pieces of the 'boundary' of $X_{0}$. Moreover, $X_{a}$ and $X_{b}$ are Cantor spaces (and $\mu$ is a Cantor measure on such spaces), and so one can easily construct measurable subsets of $X_{b}$ of arbitrary measure between 0 and $\mu\left(X_{b}\right)$. Thus Property (i) is satisfied. Furthermore, one can easily create a measure-preserving invertible map $T_{a}^{0}: X_{a} \rightarrow X_{a}$ such that $\left(T_{a}^{0}\right)^{2}$ is ergodic on $X_{a}$; this can be done for instance by identifying $X_{a}$ (which is an atomless standard probability space) as a measure space (up to null sets) with the unit circle with Haar measure, and then setting $T_{a}^{0}$ to be an irrational translation map.

We now define the shifts $T_{a}: X \rightarrow X$ and $T_{b}: X \rightarrow X$ as follows.

1. If $x \in X_{0}$, then $T_{a} x$ is defined to be $S_{a} x$ projected onto $X$, and $T_{b} x$ is similarly defined to be $S_{b} x$ projected onto $X$. (The projection is only necessary of course if $S_{a} x$ or $S_{b} x$ lands in $Y_{0}$.)

2. If $x \in X_{a}$, then $T_{a} x:=T_{a}^{0} x$. If instead $x \in X_{b}, T_{a} x$ is defined to be $S_{a} x^{\prime} \in Y_{1}$, where $x^{\prime} \in S_{a}^{-1} Y_{1} \cap Y_{0}$ is the lift of $x$ to $S_{a}^{-1} Y_{1} \cap Y_{0}$.

3. If $x \in X_{b}$, then $T_{b} x=x$. If instead $x \in X_{a}, T_{b} x$ is defined to be $S_{b} x^{\prime} \in Y_{1}$, where $x^{\prime} \in S_{b}^{-1} Y_{1} \cap Y_{0}$ is the lift of $x$ to $S_{b}^{-1} Y_{1} \cap Y_{0}$.

One then defines $T_{g}$ for the remaining $g \in F_{2}$ in the usual manner. In particular, one sees that for any $x$ in the interior $X_{0}$ and any $s \in\left\{a, b, a^{-1}, b^{-1}\right\}, T_{s} x$ is equal to $S_{s} x$ projected onto $X$. Informally, the shifts $T_{s}: X \rightarrow X$ for $s \in\left\{a, b, a^{-1}, b^{-1}\right\}$ are inherited from the shifts $S_{s}: Y \rightarrow Y$ except for the boundary actions of $T_{a}$ and $T_{a}^{-1}$ on $X_{a}$ and of $T_{b}$ and $T_{b}^{-1}$ on $X_{b}$, which are given by $T_{a}^{0}$ (and its inverse) and the identity map respectively. (There is nothing special about the identity map here; an arbitrary measure-preserving map on $X_{b}$ could be substituted here for our purposes.)

PROPOSITION 9. $\left(X, \mathcal{X}, \mu,\left(T_{g}\right)_{g \in F_{2}}\right)$ is a good system.

Proof. It is a routine matter to verify that $T_{a}$ and $T_{b}$ are invertible and measurepreserving, so that $\left(X, \mathcal{X}, \mu,\left(T_{g}\right)_{g \in F_{2}}\right)$ is an $F_{2}$-system. Property (i) was already verified. For Property (ii), we note that $T_{a} X_{b} \subset Y_{1} \subset\left(S_{b} Y_{0} \cap Y_{1}\right) \cup\left(S_{b}^{-1} Y_{0} \cap Y_{1}\right)=$ $T_{b} X_{a} \cup T_{b}^{-1} X_{a}$, as required. We set $m=1$ and $X_{a, 1}:=X_{a}$; then Property (iii) is true from construction. Finally, we verify Property (iv). It suffices to show that for almost every $x \in X$, there is a group element $g \in F_{2}$ such that $T_{g} x \in X_{a}$. This is trivial if $x \in X_{a}$, and if $x \in X_{0}$ then $x \in Y_{n}$ for some $n$, and the claim follows 
by choosing $g$ to be a suitable word of length $n$ starting with $b$ or $b^{-1}$. Finally, if $x \in X_{b}$, then by applying $T_{a}$ one can move $x$ to $Y_{1}$, at which point one can apply either $T_{b}$ or $T_{b}^{-1}$ to move $x$ to $X_{a}$.

It remains to construct a sequence $\tilde{f}_{n}$ of nonnegative functions in $L^{\infty}(\tilde{X})$ for each $n \in \mathbb{Z}$ obeying Properties (v)-(viii) with $\alpha=1$. For negative $n$, we define $\tilde{f}_{n}$ by setting

$$
\tilde{f}_{n}(x, s):=4 \times 3^{-n}
$$

whenever $x \in X$ and $s \in\left\{a, b, a^{-1}, b^{-1}\right\}$ are such that $x \in Y_{-n}$ and $S_{s} x \in Y_{-n-1}$, and $\tilde{f}_{n}(x, s)=0$ otherwise. These are clearly nonnegative functions in $L^{\infty}(\tilde{X})$ obeying Property (vii). It is routine to verify that $\tilde{f}_{n+1}=P \tilde{f}_{n}$ for all $n \leqslant-2$. If we then define $\tilde{f}_{n}$ for nonnegative $n$ by the formula

$$
\tilde{f}_{n}:=P^{n+1} \tilde{f}_{-1}
$$

then we have Property (v). For negative $n$ we have

$$
\left\|\tilde{f}_{n}\right\|_{L^{1}(\tilde{X})}=1
$$

which gives Property (vi) (using Property (v) to extend to nonnegative $n$ ). Finally, from Lemma 5 we see that $\tilde{f}_{n}$ converges pointwise almost everywhere to 1 as $n \rightarrow$ $+\infty$, and so Property (viii) follows. This concludes the proof of Theorem 7 .

\section{The iteration step}

We now prove Theorem 8 . Let $0<\alpha \leqslant 1$ be such that $P(\alpha)$ holds. Inspecting the definition of $P(\alpha)$ in Claim 6 (with $\varepsilon$ replaced by $\varepsilon / 4$ ), and normalizing $X$ to have measure 1 , we may find a good system $\left(X, \mathcal{X}, \mu,\left(T_{g}\right)_{g \in F_{2}}\right)$ with associated decomposition $X=X_{a} \cup X_{b} \cup X_{0}$ and measure $\mu(X)=1$, and a sequence of nonnegative functions $\tilde{f}_{n} \in L^{\infty}(\tilde{X})$ for $n \in \mathbb{Z}$ with the following properties:

(v) (Ancient Markov chain) $\tilde{f}_{n+1}=P \tilde{f}_{n}$ for all $n \in \mathbb{Z}$.

(vi) (Size) One has $\left\|\tilde{f}_{n}\right\|_{L^{1}(\tilde{X})}=\alpha$ for all $n \in \mathbb{Z}$.

(vii) (Early support) $\tilde{f}_{n}$ is supported in $\tilde{X}_{0}$ for all negative $n$. Furthermore, there exists a finite $A>0$ such that $\tilde{f}_{n}$ is supported in a set of measure at most $A 3^{n}$ for all negative $n$.

(viii) (Large maximum function) We have

$$
\sup _{n \in \mathbb{Z}} \pi_{*} \tilde{f}_{2 n}(x) \geqslant 1-\varepsilon / 4
$$

for all $x \in X$ outside of a set of measure at most $\varepsilon / 4$. 
It will suffice to construct a good system $\left(X^{\prime}, \mathcal{X}^{\prime}, \mu^{\prime},\left(T_{g}^{\prime}\right)_{g \in F_{2}}\right)$ with associated decomposition $X^{\prime}=X_{a}^{\prime} \cup X_{b}^{\prime} \cup X_{0}^{\prime}$, Markov operator $P^{\prime}$, and measure $\mu^{\prime}\left(X^{\prime}\right)=2$, and a sequence of nonnegative functions $\tilde{f}_{n}^{\prime} \in L^{\infty}\left(\tilde{X}^{\prime}\right)$ for $n \in \mathbb{Z}$ with the following properties:

$\left(\mathrm{v}^{\prime}\right)$ (Ancient Markov chain) $\tilde{f}_{n+1}^{\prime}=P^{\prime} \tilde{f}_{n}^{\prime}$ for all $n \in \mathbb{Z}$.

(vi') (Size) One has $\left\|\tilde{f}_{n}^{\prime}\right\|_{L^{1}\left(\tilde{X}^{\prime}\right)}=\alpha(2-\alpha / 2)$ for all $n \in \mathbb{Z}$.

(vii') (Early support) $\tilde{f}_{n}^{\prime}$ is supported in $\tilde{X}_{0}^{\prime}$ for all negative $n$. Furthermore, there exists a finite $A^{\prime}>0$ such that $\tilde{f}_{n}^{\prime}$ is supported in a set of measure at most $2 A^{\prime} 3^{n}$ for all negative $n$.

(viii') (Large maximum function) We have

$$
\sup _{n \in \mathbb{Z}} \pi_{*} \tilde{f}_{2 n}^{\prime}\left(x^{\prime}\right) \geqslant 1-\varepsilon
$$

for all $x^{\prime} \in X^{\prime}$ outside of a set of measure at most $2 \varepsilon$.

The idea is to glue together two copies of the system $X$ to form $X^{\prime}$, with an extremely small amount of coupling between the two in order to make the combined system $X^{\prime}$ ergodic. The functions $\tilde{f}_{n}^{\prime}$, for negative $n$, will consist of one copy of $\tilde{f}_{n}$ (situated in the first copy of $X$ ) and one copy of $(1-\alpha / 2) \tilde{f}_{n+M}$ (situated in the second copy of $X$ ), where $M$ is an extremely large 'time delay', designed so that the dynamics of the copy $(1-\alpha / 2) \tilde{f}_{n+M}$ only becomes 'interesting' long after the dynamics of the copy of $\tilde{f}_{n}^{\prime}$ has achieved convergence to its average on $X^{\prime}$, which will be $\alpha / 2$. It is this latter convergence to the average that allows one to place the crucial factor of $(1-\alpha / 2)$ in the second component of $\tilde{f}_{n}^{\prime}$, which leads to the gain of $\alpha$ to $\alpha(1-\alpha / 4)$ in Theorem 8 .

We turn to the details of the construction. First, from Property (viii) and Egorov's theorem, we may find a natural number $N$ such that

$$
\sup _{-N \leqslant n \leqslant N} \pi_{*} \tilde{f}_{2 n}(x) \geqslant 1-\varepsilon / 3
$$

for all $x \in X$ outside of a set of measure at most $\varepsilon / 3$. We let $0<\kappa<1 / 4$ be a small quantity depending on $\varepsilon, N$ and the $\tilde{f}_{n}$ to be chosen later. We will construct the good system $\left(X^{\prime}, \mathcal{X}^{\prime}, \mu^{\prime},\left(T_{g}^{\prime}\right)_{g \in F_{2}}\right)$ to be two copies of $\left(X, \mathcal{X}, \mu,\left(T_{g}\right)_{g \in F_{2}}\right)$ glued together by a small amount of coupling, with the $\kappa$ parameter measuring the amount of coupling. More precisely, we define the measure space $\left(X^{\prime}, \mathcal{X}^{\prime}\right.$, $\left.\mu^{\prime}\right)$ to be the product of $(X, \mathcal{X}, \mu)$ with the two-element set $\{1,2\}$ with counting measure. Next, using Property (i), we can find a subset $E$ of $X_{b}$ of measure 
exactly $\kappa$. We now define the 'shift' maps $T_{a}^{\prime}, T_{b}^{\prime}: X^{\prime} \rightarrow X^{\prime}$ as follows. The map $T_{a}^{\prime}$ is a trivial lift of $T_{a}$; thus

$$
T_{a}^{\prime}(x, i):=\left(T_{a} x, i\right)
$$

for $x \in X$ and $i \in\{1,2\}$. The map $T_{b}^{\prime}$ is an almost trivial lift of $T_{b}$. Namely, we define

$$
T_{b}^{\prime}(x, i):=\left(T_{b} x, i\right)
$$

for $x \in X \backslash E$ and $i \in\{1,2\}$, but define

$$
T_{b}^{\prime}(x, i):=\left(T_{b} x, 3-i\right)
$$

for $x \in E$ and $i \in\{1,2\}$; see Figure 3. Finally, we partition $X^{\prime}=X_{a}^{\prime} \cup X_{b}^{\prime} \cup X_{0}^{\prime}$, where $X_{a}^{\prime}:=X_{a} \times\{1,2\}, X_{b}^{\prime}:=X_{b} \times\{1,2\}$ and $X_{0}^{\prime}:=X_{0} \times\{1,2\}$. We then define $T_{g}^{\prime}$ for the remaining $g \in F_{2}$ in the usual manner.

Proposition 10 (Good system). If $\kappa$ is sufficiently small, then $\left(X^{\prime}, \mathcal{X}^{\prime}, \mu^{\prime}\right.$, $\left.\left(T_{g}^{\prime}\right)_{g \in F_{2}}\right)$ is a good system with $\mu^{\prime}\left(X^{\prime}\right)=2$.

Proof. Properties (i) and (ii) are easily verified, so we focus on verifying Properties (iii) and (iv).

By Property (iii) for $X, X_{a}$ is partitioned into finitely many $T_{a}$-invariant components $X_{a, 1}, \ldots, X_{a, m}$ of positive measure, each of which is $T_{a}^{2}$-ergodic. This induces a partition of $X_{a}^{\prime}$ into the $2 m$ components $X_{a, 1} \times\{1\}, \ldots, X_{a, m} \times\{1\}$, $X_{a, 1} \times\{2\}, \ldots, X_{a, m} \times\{2\}$, and each of these components is clearly $T_{a}^{2}$-ergodic.

Now we verify Property (iv). We need to show that $X^{\prime}=\bigcup_{g \in F_{2}} T_{g}^{\prime}\left(X_{a, i} \times\{j\}\right)$ up to null sets for each $i=1, \ldots, m$ and $j=1,2$. Denote the right-hand side by $Y$; thus $Y$ is $F_{2}$-invariant and contains $X_{a, i} \times\{j\}$. On the other hand, by Property (iv) for $X$ and the pigeonhole principle, there exists $g \in F_{2}$ such that $T_{g} X_{a, i}$ intersects $E$ in a set of positive measure. We may assume that the word length $|g|$ of $g$ is minimal among all $g$ with this property; thus $T_{h} X_{a, i} \cap E$ is null whenever $|h|<|g|$. From this we see that $T_{g}^{\prime}\left(X_{a, i} \times\{j\}\right)$ intersects $E \times\{j\}$ in a set of positive measure (since the dynamics of $T^{\prime}$ are just a trivial lift of the dynamics of $T$ outside of $E \times\{1,2\})$. From the construction of $T_{b}^{\prime}$, this implies that $T_{b g}^{\prime}\left(X_{a, i} \times\{j\}\right)$ intersects $T_{b} E \times\{3-j\} \subset X_{b} \times\{3-j\}$ in a set of positive measure, and hence by Property (ii) the union of $T_{b^{-1} a b g}^{\prime}\left(X_{a, i} \times\{j\}\right)$ and $T_{b a b g}^{\prime}\left(X_{a, i} \times\{j\}\right)$ intersects $X_{a} \times\{3-j\}$ in a set of positive measure; in particular, $Y$ intersects $X_{a} \times\{3-j\}$ in a set of positive measure. As $Y$ is $\left(T_{a}^{\prime}\right)^{2}$-invariant, we conclude from Property (iii) that $Y$ contains $X_{a, i^{\prime}} \times\{3-j\}$ up to null sets for some $i^{\prime}=1$, $\ldots, m$.

Next, by another appeal to Property (iv) and the pigeonhole principle, we can find $g_{i, i^{\prime}} \in F_{2}$ such that $T_{g_{i, i^{\prime}}} X_{a, i^{\prime}}$ and $X_{a, i}$ intersect in a set of positive measure. 


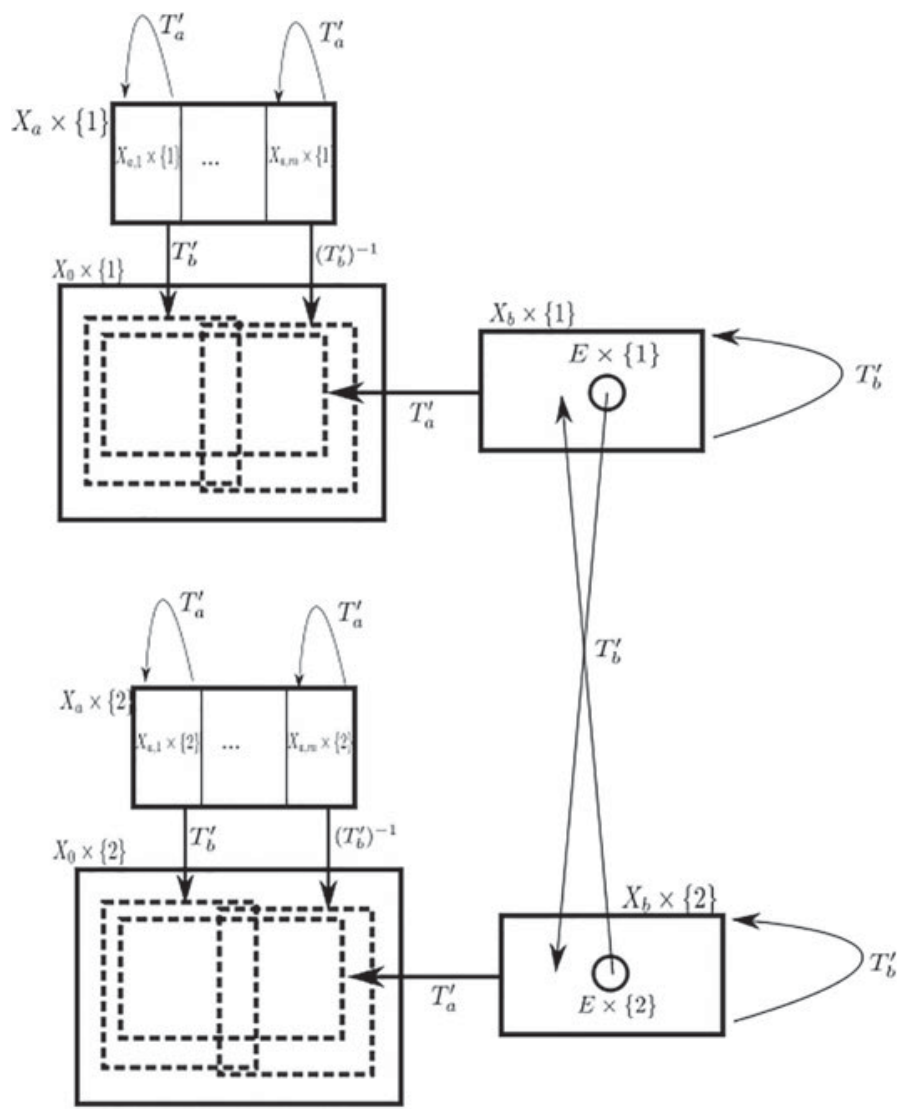

Figure 3. The good system $\left(X^{\prime}, \mathcal{X}^{\prime}, \mu^{\prime},\left(T_{g}^{\prime}\right)_{g \in F_{2}}\right)$, which is formed by gluing together two barely interacting copies of $\left(X, \mathcal{X}, \mu,\left(T_{g}\right)_{g \in F_{2}}\right)$.

Note that as there are only $m$ choices for $i^{\prime}$, the word length of $g_{i, i^{\prime}}$ can be bounded above, and the measure of $T_{g_{i, i}} X_{a, i^{\prime}} \cap X_{a, i}$ bounded below, by quantities independent of $\kappa$. Because of this, we see that if $\kappa$ (and hence $E$ ) is small enough, then $T_{g_{i, i^{\prime}}}^{\prime}\left(X_{a, i^{\prime}} \times\{3-j\}\right)$ and $X_{a, i} \times\{3-j\}$ also intersect in a set of positive measure; thus $Y$ must intersect $X_{a, i} \times\{3-j\}$ in a set of positive measure, and hence by the $T_{a}^{2}$-ergodicity of $X_{a, i}, Y$ contains $X_{a, i} \times\{3-j\}$ up to null sets. Since $Y$ already contained $X_{a, i} \times\{j\}$, we have $X_{a, i} \times\{1,2\}$ contained in $Y$ up to null sets.

Now for any $\left(x, j^{\prime}\right) \in X^{\prime}$, we have from Property (iv) that $x=T_{g} y$ for some $y \in X_{a, i}$ and $g \in F_{2}$. This implies that $\left(x, j^{\prime}\right)=T_{g}^{\prime}\left(y, j^{\prime \prime}\right)$ for some $j^{\prime \prime} \in\{1,2\}$, and hence $\left(x, j^{\prime}\right) \in Y$ for almost every $\left(x, j^{\prime}\right) \in X$, which gives Property (iv) for $X^{\prime}$ as required. 
We let $M$ be a large natural number, depending on all previous quantities (in particular, depending on $\kappa$ ), to be chosen later. The functions $\tilde{f}_{n}^{\prime} \in L^{1}\left(\tilde{X}^{\prime}\right)$ will be defined for negative $n$ by the formulas

$$
\tilde{f}_{n}^{\prime}(x, 1, s):=\tilde{f}_{n}(x, s)
$$

and

$$
\tilde{f}_{n}^{\prime}(x, 2, s):=\left(1-\frac{\alpha}{2}\right) \tilde{f}_{n-2 M}(x, s)
$$

for any $x \in X$ and $s \in\left\{a, b, a^{-1}, b^{-1}\right\}$. Informally, $\tilde{f}_{n}^{\prime}$ is two copies of $\tilde{f}_{n}^{\prime}$, one over $X \times\{1\}$ and one over $X \times\{2\}$, with the latter experiencing a significant time delay and also a slight reduction in amplitude; the point is that we can delay the $X \times\{2\}$ dynamics until the dynamics of $X \times\{1\}$ have mixed almost completely, so that half of the mass of the $X \times\{1\}$ component is spread out almost uniformly over $X \times\{2\}$, allowing for the crucial amplitude reduction for the $X \times\{2\}$ component. The idea behind this construction is due to Ornstein [12, Lemma 4].

Clearly, Property (vii') is a consequence of Property (vii) (we allow the constant $A^{\prime}$ to depend on $M$ ). For functions supported on $\tilde{X}_{0}^{\prime}$, the Markov operator $P^{\prime}$ is a trivial lift of the Markov operator $P$, so (from Property (vii')) one sees that $\tilde{f}_{n+1}^{\prime}=P^{\prime} \tilde{f}_{n}^{\prime}$ for all $n \leqslant-2$. We now define $\tilde{f}_{n}^{\prime}$ for nonnegative $n$ by setting

$$
\tilde{f}_{n}^{\prime}:=\left(P^{\prime}\right)^{n+1} \tilde{f}_{-1}^{\prime},
$$

so that Property $\left(\mathrm{v}^{\prime}\right)$ holds. Clearly $\tilde{f}_{n}^{\prime}$ are nonnegative and in $L^{\infty}$, and direct calculation shows that Property (vi') holds for all negative $n$, and hence for all $n$ thanks to Property $\left(\mathrm{v}^{\prime}\right)$.

The only remaining task is to show Property (viii'). By the union bound, it suffices to show the bounds on $X \times\{1\}$ and $X \times\{2\}$ separately. More precisely, we will show (for suitable choices of the $\kappa$ and $M$ parameters) that

$$
\sup _{n \in \mathbb{Z}} \pi_{*} \tilde{f}_{2 n}^{\prime}(x, 1) \geqslant 1-\varepsilon
$$

for all $x \in X$ outside of a set of measure at most $\varepsilon$, and

$$
\sup _{n \in \mathbb{Z}} \pi_{*} \tilde{f}_{2 n}^{\prime}(x, 2) \geqslant 1-\varepsilon
$$

for all $x \in X$ outside of a set of measure at most $\varepsilon$.

We split

$$
\tilde{f}_{n}^{\prime}=\tilde{f}_{n, 1}^{\prime}+\tilde{f}_{n, 2}^{\prime}
$$

where for negative $n, \tilde{f}_{n, i}^{\prime}$ is the restriction of $\tilde{f}_{n}^{\prime}$ to $X \times\{i\} \times\left\{a, b, a^{-1}, b^{-1}\right\}$, and for nonnegative $n, \tilde{f}_{n, i}^{\prime}$ is propagated by $P^{\prime}$ :

$$
\tilde{f}_{n, i}^{\prime}:=\left(P^{\prime}\right)^{n+1} \tilde{f}_{-1, i}^{\prime}
$$


Observe (by induction on $n$ in the nonnegative case) that the $\tilde{f}_{n, 1}^{\prime}$ component of $\tilde{f}_{n}^{\prime}$ is nonnegative and does not depend on $M$, and the $\tilde{f}_{n, 2}^{\prime}$ component is related to the $\tilde{f}_{n, 1}^{\prime}$ component by the formula

$$
\tilde{f}_{n, 2}^{\prime}(x, i)=\left(1-\frac{\alpha}{2}\right) \tilde{f}_{n-M, 1}^{\prime}(x, 3-i) .
$$

We first prove a bound on $\tilde{f}_{n, 1}^{\prime}$ :

Proposition 11 (Bound on $\tilde{f}_{n, 1}^{\prime}$ ). If $\kappa$ is sufficiently small (depending on $\varepsilon, N$, and $\tilde{f}_{n}$, but without any dependence on $M$ ), we have

$$
\sup _{-N \leqslant n \leqslant N} \pi_{*} \tilde{f}_{2 n, 1}^{\prime}(x, 1) \geqslant 1-2 \varepsilon / 3
$$

for all $x \in X$ outside of a set of measure at most $2 \varepsilon / 3$.

Proof. By construction, we have

$$
\tilde{f}_{n, 1}^{\prime}(x, 1, s)=\tilde{f}_{n}(x, s)
$$

for negative $n$, all $x \in X$, and $s \in\left\{a, b, a^{-1}, b^{-1}\right\}$. Now we turn to nonnegative $n$. Note that as $P$ is a contraction on $L^{\infty}, \tilde{f}_{n}$ for nonnegative $n$ are uniformly bounded in $L^{\infty}$ by some quantity $B$ independent of $\kappa$. A routine induction then shows that

$$
\int_{\tilde{X}} \max \left(\tilde{f}_{n}(x, s)-\tilde{f}_{n, 1}^{\prime}(x, 1, s), 0\right) d \tilde{\mu}(x, s) \leqslant C_{B, n} \kappa
$$

for all nonnegative $n$ and some quantity $C_{B, n}$ that depends on $B, n$ but not on $\kappa$; this is basically because on $X \times\{1\} \times\left\{a, b, a^{-1}, b^{-1}\right\}$, the Markov process associated with $P^{\prime}$ only differs from that associated with $P$ on the set $E \times\{1\} \times$ $\{b\} \cup T_{b} E \times\{1\} \cup\left\{b^{-1}\right\}$, which has measure $\kappa / 2$. Applying $\pi_{*}$ and then the triangle inequality, we conclude that

$$
\int_{X} \max \left(\sup _{-N \leqslant n \leqslant N} \pi_{*} \tilde{f}_{2 n}(x)-\sup _{-N \leqslant n \leqslant N} \pi_{*} \tilde{f}_{2 n, 1}^{\prime}(x, 1), 0\right) d \mu(x) \leqslant C_{B, N}^{\prime} \kappa
$$

for some $C_{B, N}^{\prime}$ independent of $\kappa$; in particular, from Markov's inequality we see (for $\kappa$ small enough) that

$$
\sup _{-N \leqslant n \leqslant N} \pi_{*} \tilde{f}_{2 n}(x)-\sup _{-N \leqslant n \leqslant N} \pi_{*} \tilde{f}_{2 n, 1}^{\prime}(x, 1) \leqslant \varepsilon / 3
$$

for all $x \in X$ outside of a set of measure at most $\varepsilon / 3$. Combining this with (1), we obtain the claim. 
Henceforth we take $\kappa$ small enough so that Proposition 11 holds; note crucially that we do not need $\kappa$ to depend on $M$ so that $M$ is still free to be arbitrarily large; in particular, we may take $M$ to be larger than any given function of $\kappa$.

From (4) we have the pointwise bound

$$
\pi_{*} \tilde{f}_{2 n}^{\prime}(x, 1) \geqslant \pi_{*} \tilde{f}_{2 n, 1}^{\prime}(x, 1)
$$

and so the desired bound (2) follows from Proposition 11.

It remains to establish (3). From (5) and Proposition 11, we have

$$
\sup _{M-N \leqslant n \leqslant M+N} \pi_{*} \tilde{f}_{2 n, 2}^{\prime}(x, 2) \geqslant 1-\frac{\alpha}{2}-2 \varepsilon / 3
$$

for all $x \in X$ outside of a set of measure at most $2 \varepsilon / 3$ (we have discarded the small additional term of $\alpha \varepsilon / 3$ on the right-hand side).

This by itself is not yet enough to establish (3). However, from Lemma 5, we see that $\tilde{f}_{n, 1}^{\prime}$ converges pointwise almost everywhere as $n \rightarrow \infty$ to the constant

$$
\frac{1}{\mu\left(\tilde{X}^{\prime}\right)} \int_{\tilde{X}^{\prime}} \tilde{f}_{-1,1}^{\prime} d \tilde{\mu}^{\prime}=\frac{1}{2} \int_{X} \tilde{f}_{-1} d \tilde{\mu}=\frac{\alpha}{2} .
$$

In particular, $\pi_{*} \tilde{f}_{n, 1}^{\prime}$ converges pointwise almost everywhere to $\alpha / 2$. Thus, by Egorov's theorem, and assuming $M$ sufficiently large (depending on previous quantities such as $\varepsilon, \kappa$, and $\tilde{f}_{n}$, but without any circular dependency of $M$ on itself) we have

$$
\inf _{n \geqslant M-N} \pi_{*} \tilde{f}_{2 n, 1}^{\prime}(x, 2) \geqslant \frac{\alpha}{2}-\frac{\varepsilon}{3}
$$

for all $x \in X$ outside of a set of measure at most $\varepsilon / 3$. Adding this to (6) and using (4), we obtain (3) as required.

The proof of Theorem 8, and thus Theorem 2, is now complete.

\section{Acknowledgements}

The author is supported by the National Science Foundation (NSF) grant DMS1266164 and by a Simons Investigator Award, and thanks Lewis Bowen for helpful discussions and corrections. Finally, the author thanks the anonymous referee for suggestions and corrections.

\section{References}

[1] L. Bowen and A. Nevo, 'Pointwise ergodic theorems beyond amenable groups', Ergodic Theory Dynam. Systems 33(3) (2013), 777-820.

[2] L. Bowen and A. Nevo, 'Amenable equivalence relations and the construction of ergodic averages for group actions', J. Anal. Math. 126 (2015), 359-388. 
[3] A. Bufetov, 'Convergence of spherical averages for actions of free groups', Ann. of Math. (2) 155(3) (2002), 929-944.

[4] A. I. Bufetov and A. V. Klimenko, 'Maximal inequality and ergodic theorems for Markov groups', Proc. Steklov Inst. Math. 277 (2012), 27-42.

[5] A. I. Bufetov, M. Khristoforov and A. Klimenko, 'Cesàro convergence of spherical averages for measure-preserving actions of Markov semigroups and groups', Int. Math. Res. Not. IMRN 2012(21) (2012), 4797-4829.

[6] K. Fujiwara and A. Nevo, 'Maximal and pointwise ergodic theorems for word-hyperbolic groups', Ergodic Theory Dynam. Systems 18(4) (1998), 843-858.

[7] E. Lindenstrauss, 'Pointwise theorems for amenable groups', Invent. Math. 146(2) (2001), 259-295.

[8] G. A. Margulis, A. Nevo and E. Stein, 'Analogs of Wiener's ergodic theorems for semisimple Lie groups. II', Duke Math. J. 103(2) (2000), 233-259.

[9] A. Naor and T. Tao, 'Random martingales and localization of maximal inequalities', J. Funct. Anal. 259(3) (2010), 731-779.

[10] A. Nevo and E. Stein, 'A generalization of Birkhoff's pointwise ergodic theorem', Acta Math. 173(1) (1994), 135-154.

[11] A. Nevo and E. Stein, 'Analogs of Wiener's ergodic theorems for semisimple groups. I', Ann. of Math. (2) 145(3) (1997), 565-595.

[12] D. Ornstein, 'On the pointwise behavior of iterates of a self-adjoint operator', J. Math. Mech. $18(1968 / 1969), 473-477$.

[13] G.-C. Rota, 'An 'Alternierende Verfahren' for general positive operators', Bull. Amer. Math. Soc. 68 (1962), 95-102.

[14] E. M. Stein, 'On the maximal ergodic theorem', Proc. Natl. Acad. Sci. USA 47 (1961), 1894-1897. 